\title{
Chromatin Interaction Analysis with Paired-End Tag (ChIA-PET) sequencing technology and application
}

\author{
Guoliang Li ${ }^{\text {* }}$, Liuyang Cai ${ }^{1}$, Huidan Chang ${ }^{1}$, Ping Hong ${ }^{1}$, Qiangwei Zhou', Ekaterina V Kulakova ${ }^{2,3}$, \\ Nikolay A Kolchanov ${ }^{2,3}$, Yijun Ruan ${ }^{4}$ \\ From IX International Conference on the Bioinformatics of Genome Regulation and StructurelSystems \\ Biology (BGRSISB-2014) \\ Novosibirsk, Russia. 23-28 June 2014
}

\begin{abstract}
Background: Long-range chromatin interactions play an important role in transcription regulation. Chromatin Interaction Analysis with Paired-End-Tag sequencing (ChIA-PET) is an emerging technology that has unique advantages in chromatin interaction analysis, and thus provides insight into the study of transcription regulation.

Results: This article introduces the experimental protocol and data analysis process of ChIA-PET, as well as discusses some applications using this technology. It also unveils the direction of future studies based on this technology.

Conclusions: Overall we show that ChIA-PET is the cornerstone to explore the three-dimensional (3D) chromatin structure, and certainly will lead the forthcoming wave of 3D genomics studies.
\end{abstract}

\section{Background}

Transcription regulation is a complex yet well-organized process in eukaryotes, in which chromatin interactions play a critical role and thus serve to regulate gene expression as well as further influence other cellular activities. Many technologies have been developed to study the binding of transcription factors (TF) for transcription regulation, such as chromatin immunoprecipitation (ChIP) microarray (ChIP-chip) [1], ChIP-PET [2] and ChIP-Seq [3], but they are unable to determine the target genes of the distal TF binding sites. Another challenge is to define whether such distal binding sites are functional, i.e. physically proximal to target gene promoters via chromosome loops or attracting RNA polymerase II complex for gene transcription. Therefore, identification of genome-wide distal chromatin interactions that lead the regulatory elements to their target genes may provide novel insights into the study of transcription regulation. Chromosome

\footnotetext{
* Correspondence: guoliang.li@mail.hzau.edu.cn

${ }^{1}$ National Key Laboratory of Crop Genetic Improvement, College of Informatics, Huazhong Agricultural University, 1, Shizishan Street, Wuhan, 430070, China

Full list of author information is available at the end of the article
}

conformation capture (3C) [4] and its derivatives, 4C [5,6] and $5 \mathrm{C}$ [7] can reveal long-range chromatin interactions involved in transcription regulation, but these techniques are limited either because they are low-throughout, such as $3 \mathrm{C}$, or they can't map interacting regions with high resolution in the whole genome [8] (Figure 1). In this case, it is desired to have a method capable of analyzing chromatin interactions on genome level with high throughput and high resolution.

Chromatin Interaction Analysis with Paired-End-Tag sequencing (ChIA-PET) method [9] fits these demands. It is an unbiased, genome-wide, high-throughput and de novo method. Compared with $\mathrm{Hi}-\mathrm{C}$ [10], another emerging method for chromatin interactions at a global scale, ChIA-PET is better at its higher resolution associated with a protein of interest for functional study, and lays a solid foundation for studying long-range chromatin interactions in a three-dimensional (3D) manner, as well as provides a more reliable way to determine TF binding sites and identify chromatin interactions.

Till now, ChIA-PET has been successfully applied to human MCF7 cells [9], human cancer cells [11], human $\mathrm{T}$ cells [12], mouse embryonic stem cells [13-15], mouse 


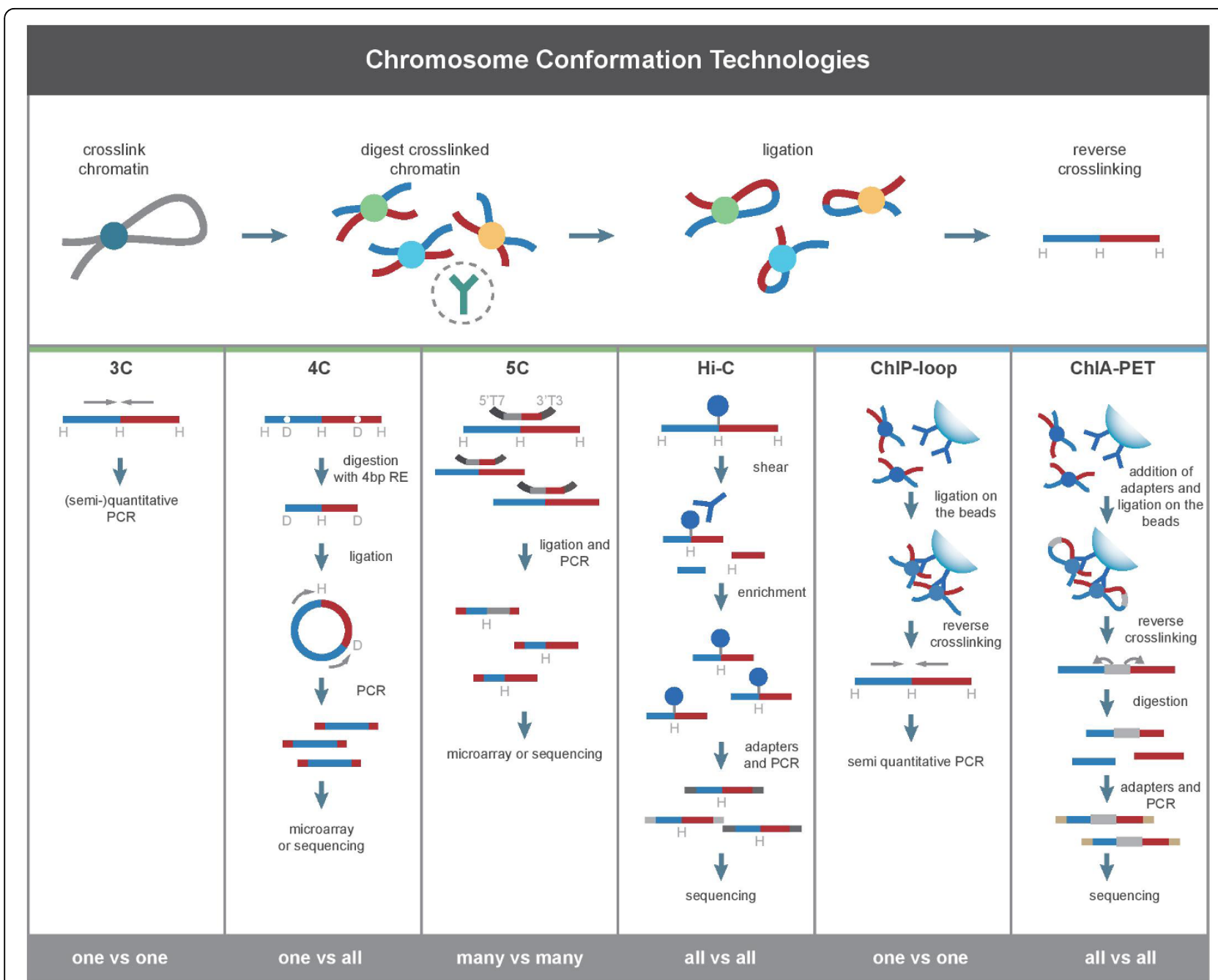

Figure 1 Comparison among 3C and its derived methods. This figure is from De Wit and de Laat, 2012 [8].

neural progenitor cells [15], and mouse B cells [16], and other cells [17-19], referring to Additional file 1 Table 1 for the available list of ChIA-PET applications by our best knowledge. Li et al. [11] has identified three kinds of interactions, named enhancer-promoter, enhancerenhancer and promoter-promoter interactions, and demonstrated that over $40 \%$ of enhancers don't regulate their nearest promoters. Sandhu et al. [20] has proposed the concept of "chromatin interaction networks", showing the phenomenon that human genome converges to a scale-free and hierarchical network, through which functions are enriched in the so-called "chromatin communities". This work unveiled the chromatin interactions in high order architectures; these architectures may act as transition from linear map to 3D/4D genome studies. Several protein associated interactions like ER$\alpha$, RNA polymerase II (RNAPII), CTCF and SMCIA have been studied to investigate how remote regulators interact with their target promoters.
In this paper, we will introduce the experimental protocol and data analysis procedure of ChIA-PET technology in comparison with other genome methods for chromosome conformation studies, and discuss the applications of ChIA-PET technology on different proteins and human/ mouse cells.

\section{Experimental process}

Compared with other $3 \mathrm{C}$-derived technologies, ChIA-PET protocol is a complex process. It can be summarized into three parts: wet-lab experiments (Figure 2), data analysis (dry-lab experiments, Figure 3 ) and experimental verification.

First, the ChIA-PET wet lab complies with the ChIP experiment [21]. Like ChIP-Seq experiment, formaldehyde is used to crosslink DNA-protein complexes in the nucleus and followed by breaking the complexes into fragments with sonication. Then, ChIP is used to enrich DNA fragments bound by a protein of interest. Next, 


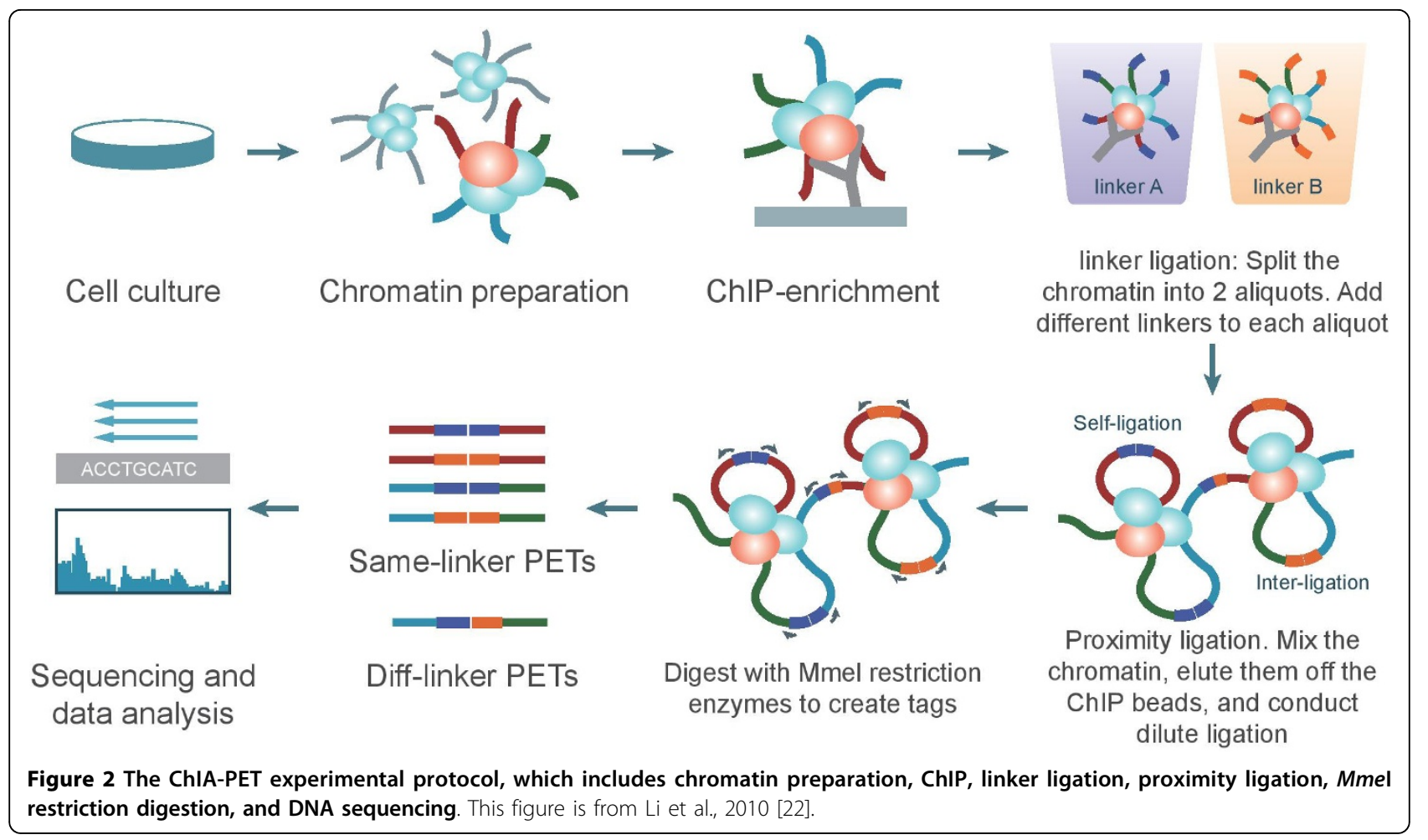

DNA fragments in ChIP-enriched chromatin complexes are ligated with two different half-linker oligonucleotides

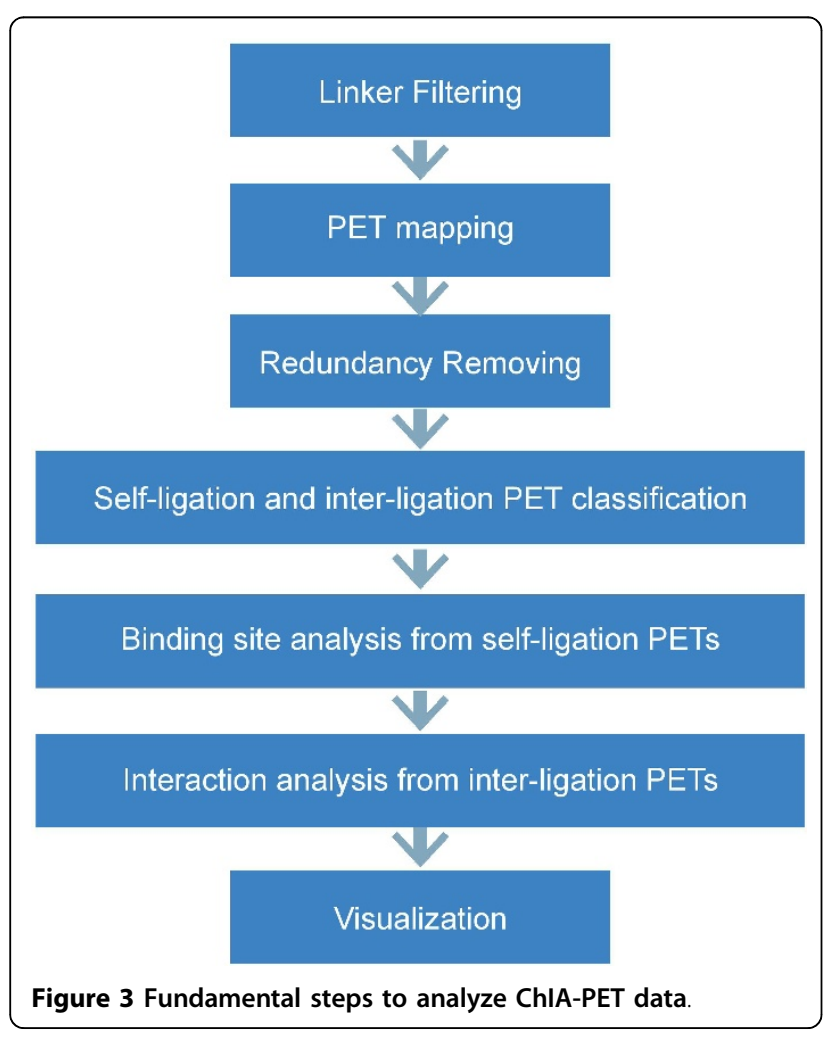

in two aliquots. Then, the two aliquots are mixed and proximal half-linkers would be ligated with each other. After reverse crosslinking, the proteins in the complexes are digested and the DNA fragments are extracted. After digestion with restriction enzyme $M m e I$, DNA fragments form paired-end tags (PETs) constructs, in "tag-linker-tag" order. Eventually, the PETs are taken to sequencing with new-generation sequencing facilities, like Illumina $\mathrm{Hi}$ Seq2500. The sequence reads are aligned to the reference genome and further analyses are performed to reveal longrange interactions between functional elements. During the whole experiments, the quality of DNA that is enriched by ChIP is the critical part. And the essence of ChIA-PET is the linker's design. The linkers not only have MmeI restriction site that could cut 20 bp outward, but also have barcodes to estimate the noise caused by random ligation.

The results of pair-end sequencing are stored in two fastq files, which can be processed with ChIA-PET Tool [22] or other methods [23]. Generally, there are seven steps in ChIA-PET data processing (Figure 3): 1) linker filtering, 2) PET mapping, 3) redundancy removing, 4) self-ligation and inter-ligation PET classification, 5) binding site analysis with self-ligation PETs, 6) chromatin interaction analysis with inter-ligation PETs, and 7) visualization of the chromatin interaction data. In the first step, linkers will be aligned to the reference halflinker nucleotide sequences. There are two kinds of 
half-linkers, named as A and B, which have the same nucleotides, except the barcodes. Therefore, PETs are classified into two categories based on the linker compositions: same linkers (AA or $\mathrm{BB}$ ) and different linkers ( $A B$ or $B A)$. Then linkers are excluded from the raw reads and the remaining DNA fragments are kept for further analysis. After linker filtering, the short DNA sequences are aligned to reference genome using BWA [24], Bowtie [25], BatMis [26], or other mapping tools. With SAMtools [27] and BEDtools [28], the redundant and low quality mapping sequences are filtered out. Next, PETs can be divided into self-ligation PETs and inter-ligation PETs. Self-ligation PETs refer to the reads from individual DNA fragments looping through their both ends and are mapped to genome within a short distance on the same chromosome. Inter-ligation PETs refer to the reads from different DNA fragments, and are generally with both tags mapped in different chromosomes or in long distance in the same chromosome. While self-ligation PETs are used to figure out protein binding sites on the genome, inter-ligation PETs can predict the chromatin interactions by clustering. Then we also have to make sure the interaction clusters between two anchors really exist or just occur by chance. Li et al. [22] used Fisher's exact test based on hypergeometric distribution to quantify the interaction frequency. Recently, Paulsen et al. [23] proposed a new statistical model based upon non-central hypergeometric distribution, which takes genome distancedependent relationships into account for $\mathrm{p}$-value estimation. Finally, ChIA-PET browser can be built to report data and visualize binding sites as well as interaction clusters.

The interactions obtained by data processing require validation with wet-lab experiment. The interactions between DNA elements in short genomic distance can be validated through $3 \mathrm{C}$ experiment. As for DNA fragments in long-range interactions (two anchors located in different chromosomes or in the same chromosome with more than 1 million base pair away), we could use microscope technique like DNA Fluorescence in situ hybridization (DNA-FISH) [29] to directly observe the location of interaction anchors and relative spatial distance in the nucleus.

\section{Applications of ChIA-PET technology} Interactions between DNA elements mediated by TFs

While ChIP-Seq is used to analyze the interactions between DNA and protein, ChIA-PET works on the interactions between DNA fragments fundamentally. Fullwood et al. [9] used ChIA-PET technology to construct chromatin interaction network bound by estrogen receptor $\alpha$ (ER- $\alpha)$ from human breast cancer cell line MCF7 and found long-range ER- $\alpha$ binding sites are mostly located at promoter regions. Handoko et al. [13] found the CTCF-mediated interactions from mouse embryonic pluripotent stem cells. Five distinct chromatin domains revealed by CTCF ChIA-PET raised a new model of CTCF function for chromosome structure organization and linking enhancers to promoters for gene transcription regulation. Li et al. [11] detected promoter-centered distant interactions bound by RNAPII in cancer cells. In addition to promoter-enhancer and enhancer-enhancer interactions, they found that promoter-promoter interactions are also pervasive in human cells. In all the promoter-nonpromoter interactions, more than $40 \%$ of the non-promoter regulatory elements didn't interact with their nearest promoters. This means that the current assumption in ChIP-Seq study the transcription factor binding sites regulate their nearest genes - is not valid. Based on the RNAPII-mediated chromatin interactions, especially promoter-promoter interactions, they proposed three types of transcription models (Figure 4): 1) basal promoter models, in which the gene promoter regions are enriched with RNAPII, but are not involved with any chromatin interactions; 2 ) single-gene interaction models, in which one gene is involved with one or more promoter-nonpromoter interactions; and 3) multi-gene interaction models, in which multiple genes are linked together by chromatin interactions to form a transcription factory for potential correlated transcription. Zhang et al. [15] figured out the interactions mediated by RNAPII in mouse pluripotent embryonic stem cells, neural stem cells and neural progenitor cells. 40,000 long-range interactions from their work uncovered the associations between promoters and distal-acting enhancers. Demare et al. [18] first described cohesin-associated chromatin interactions on the genome-wide study by ChIA-PET. $65 \%$ of interactions bound by cohesin subunit SMC1A are co-occupied by CTCF. Analyzing the interactions in multiple tissues, they proposed a potential model of tissue-specific gene regulation.

After characterizing enhancer-promoter interactions in human $\mathrm{T}$ cells, Chepelev et al. [12] presented that enhancers increase the expression of their target genes in a cell-specific way, and interacting promoters are coexpressed. In addition, chromosomes in nucleus are organized in multiple levels to perform functions, and many factors besides CTCF may be involved in this process in $\mathrm{T}$ cells. The detailed mechanism needs to be addressed in future studies.

He et al. [30] created an efficient "classifier", calculating the possibility of DNA looping based on ER- $\alpha$ binding peaks obtained by ChIP-Seq, and then predicted chromatin interactions mediated by ER- $\alpha$. This is the first work using ChIP-Seq to predict chromatin interactions, providing a supplement to ChIA-PET. 


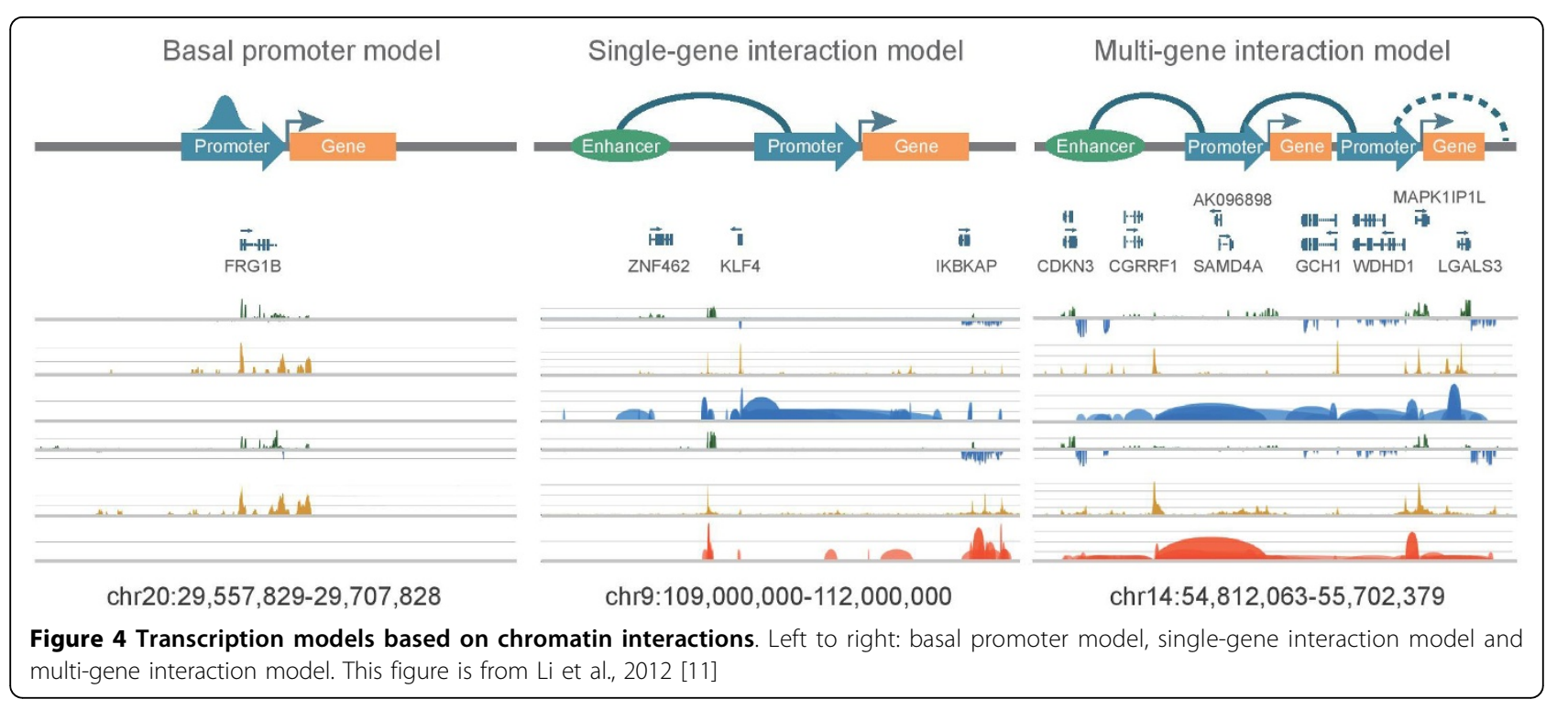

\section{Chromatin interaction network}

Cell is a complex system, and its functional characteristics rely on the cross talks of constituents including DNA, RNA, proteins, etc. Biological scientists have tried to use network approaches to build frameworks of these interactions. Many biological networks, such as metabolic networks, protein-protein interaction networks have been widely studied. However, networks of chromatin interactions are not known till recently because the lack of proper genome-wide datasets. Now data generated by ChIA-PET is unfolding the study of chromatin interaction networks.

Anchors in different interacting pairs are not isolated they may overlap with or link to other counterparts in different physical regions. This leads to the concept of "chromatin interaction networks" [31], which refers to the 2D structure of genome-wide chromatin interaction (Figure 5). Like many cellular networks, chromatin interaction network [20] has scale-free and modular topology - most nodes participate in only one or two interactions, while a few nodes, known as "hubs", connect with a disproportionately high number of nodes. These "hubs" prefer to link to each other and conform "rich-club", which carry out essential cellular functions and contribute to the robustness of the network. Chromatin interaction network is organized into "community", and genes within community perform related functions and respond to external stimuli in a coordinated manner, which means these communities may have been shaped during millions of years' evolution.

This approach has also been applied to interactions between microRNA genes. MicroRNAs are small noncoding RNAs that play a key role in transcription regulation. Chen et al. [32] has built chromatin interaction networks involving both microRNAs and protein-coding genes. The network consists of 2292 communities, and these communities are enriched in fundamental cellular

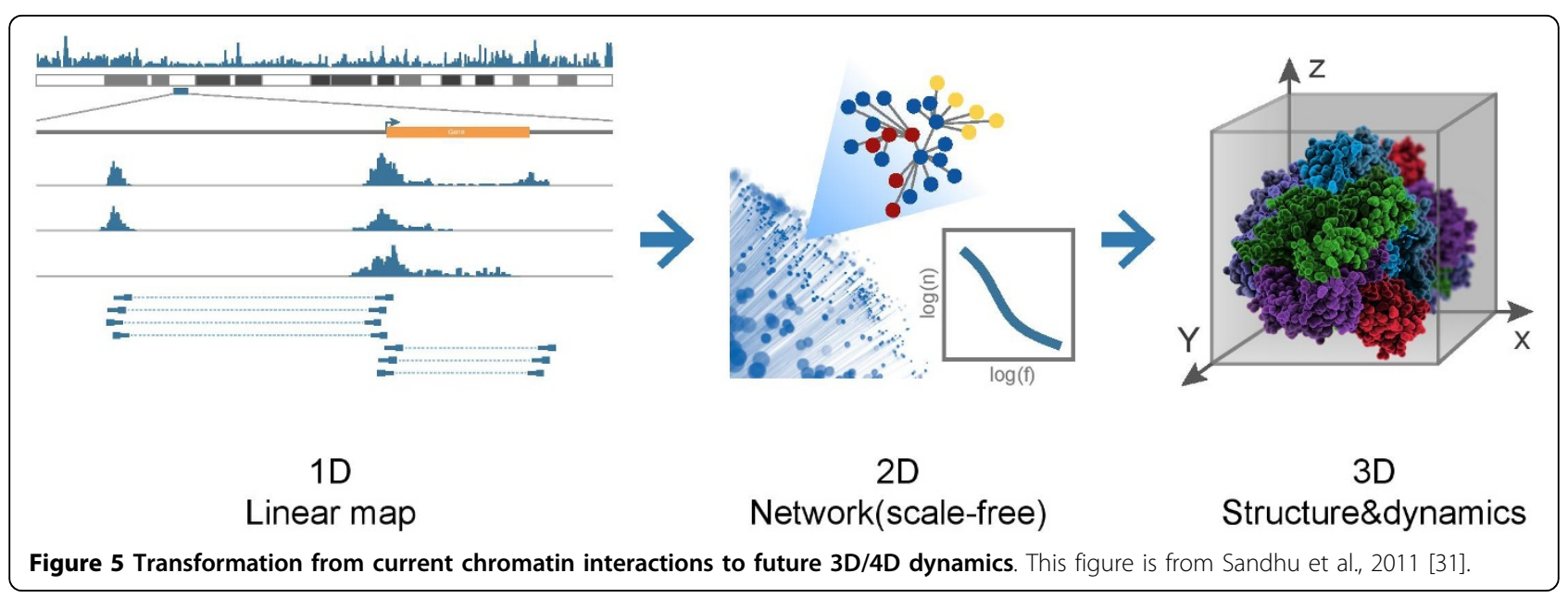


functions. The disease-related microRNAs tend to interact with each other.

In future studies, we can not only apply the method to other specific kinds of genes, but also combine interdependent networks, since cellular activities happen together and link to each other. Also, chromatin interaction networks may lay the foundation of 3D or even 4D genome waves, which transfer from static to dynamic [31].

\section{Functional studies of chromatin interactions}

There are several ways to study the functions of chromatin interactions identified by ChIA-PET: 1) luciferase reporter gene assay [11]; 2) knock-down experiment for the expression level of the protein of interest [11];3) enhancer assay from transgenic experiments for the identified regulatory elements [15]; and 4) genomic editing methods (such as zinc-finger nuclease genome editing, TALENs and CRISPR/Cas9) to perturb the chromatin interactions [16]. We will take the luciferase reporter gene assay and the TALENs as examples.

In Figure 6, ChIA-PET data shows that there are chromatin interactions between promoter regions of gene C14orf102 and CALM1, and also there are interactions between a distal regulatory element and CALM1

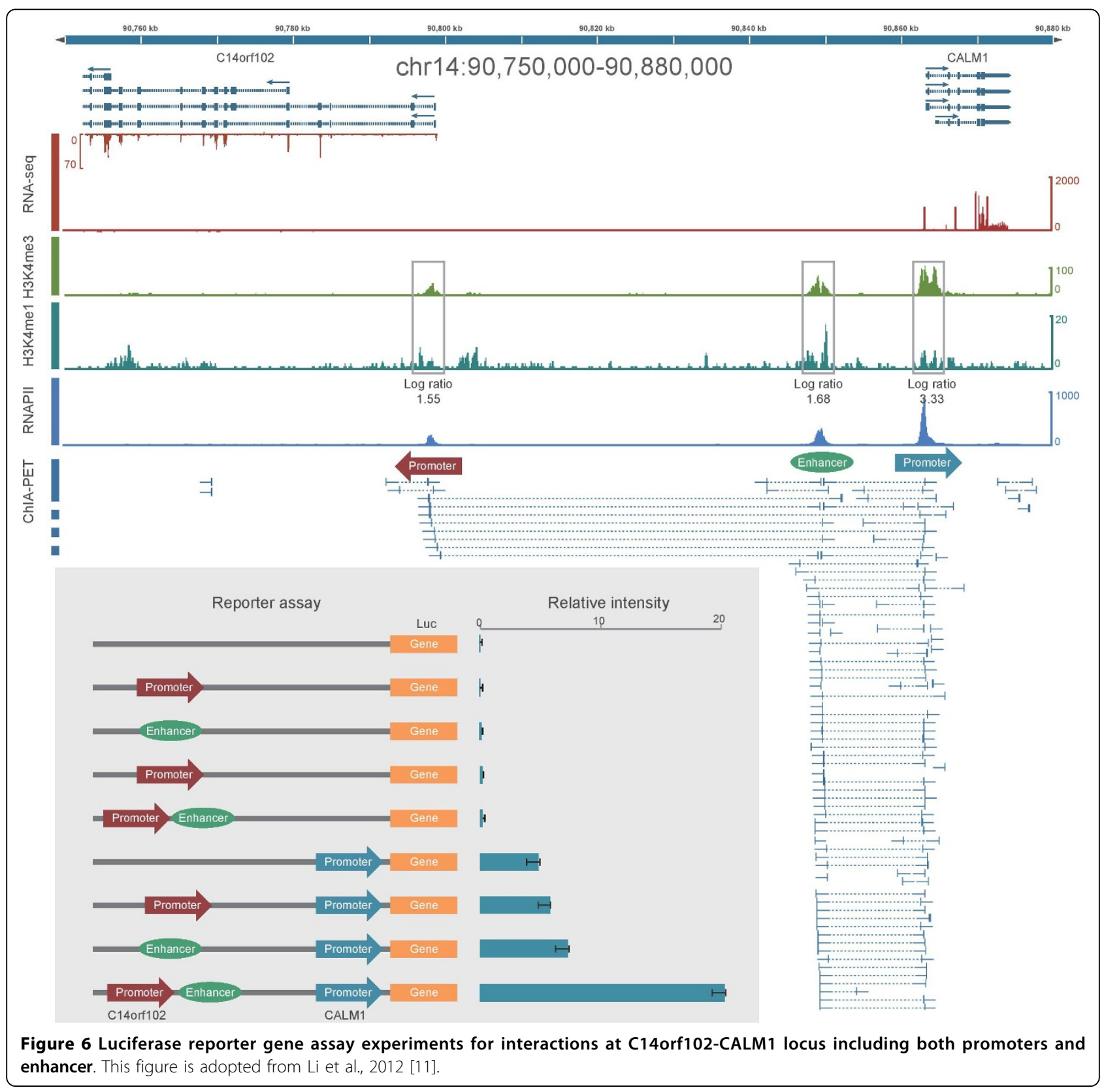


promoter. The distal regulatory element is categorized as an enhancer because it is enriched with histone mark H3K4me1. This means that the CALM1 promoter interacts with two other regions: C14orf102 promoter region and the distal enhancer. The luciferase reporter gene assay with interaction anchors shows that 1) without promoter, the luciferase reporter assay only has marginal expression level, with/without other regulatory elements; 2) with CALM1 promoter sequence alone, the luciferase reporter assay has basal expression level; 3) with promoter and one of the regulatory elements, the expression level in the luciferase reporter assay only increases marginally; and 4) with both regulatory elements, the expression level in the luciferase assay increases more than 3 folds compared with the expression level from assay with promoter alone. This means that there are combinatorial effects in the regulatory elements for gene expression regulation [11].

In Figure 7, the upper panel shows that there are six enhancer regions linked to the promoter region of gene Aicda in mouse B cells, and the enhancer E2 was selected as the region to knock out by TALEN experiment. The lower panel shows that the RNAPII and Nipbl binding intensities at the Aicda promoter region are much reduced in the genetically modified cells, compared to the binding intensities in the wild-type cells. This means that the enhancer E2 really influence the transcription regulation of gene Aicda.

\section{Reconstruction of the 3D structure of chromatin}

A precise three-dimensional structure of chromatin provides a better landscape of the biological functions. So far, the data of remote interaction is suitable to reconstruct the $3 \mathrm{D}$ genome structure. Two $3 \mathrm{C}$ derivations, namely $\mathrm{Hi}-$ C [10] and ChIA-PET [9], actually reflect the structure of the whole genome. The Hi-C technology could capture all the interactions but with low resolution. The ChIA-PET technology greatly enhances the resolution but it can only identify the interactions mediated by a known protein. So, ChIA-PET data can be used to conduct more intense modeling.

Much progress has been made in building 3D chromosomal structure from $\mathrm{Hi}-\mathrm{C}$ data [33-35]. However, there is no published article to build 3D genome structure from ChIA-PET data yet. But some research institutions try to work on it. The methods to reconstruct the 3D structure with chromatin interaction data are based on the assumption that, if the interaction frequency between two loci is high, the spatial distance between the two loci is proximal and vice versa. There

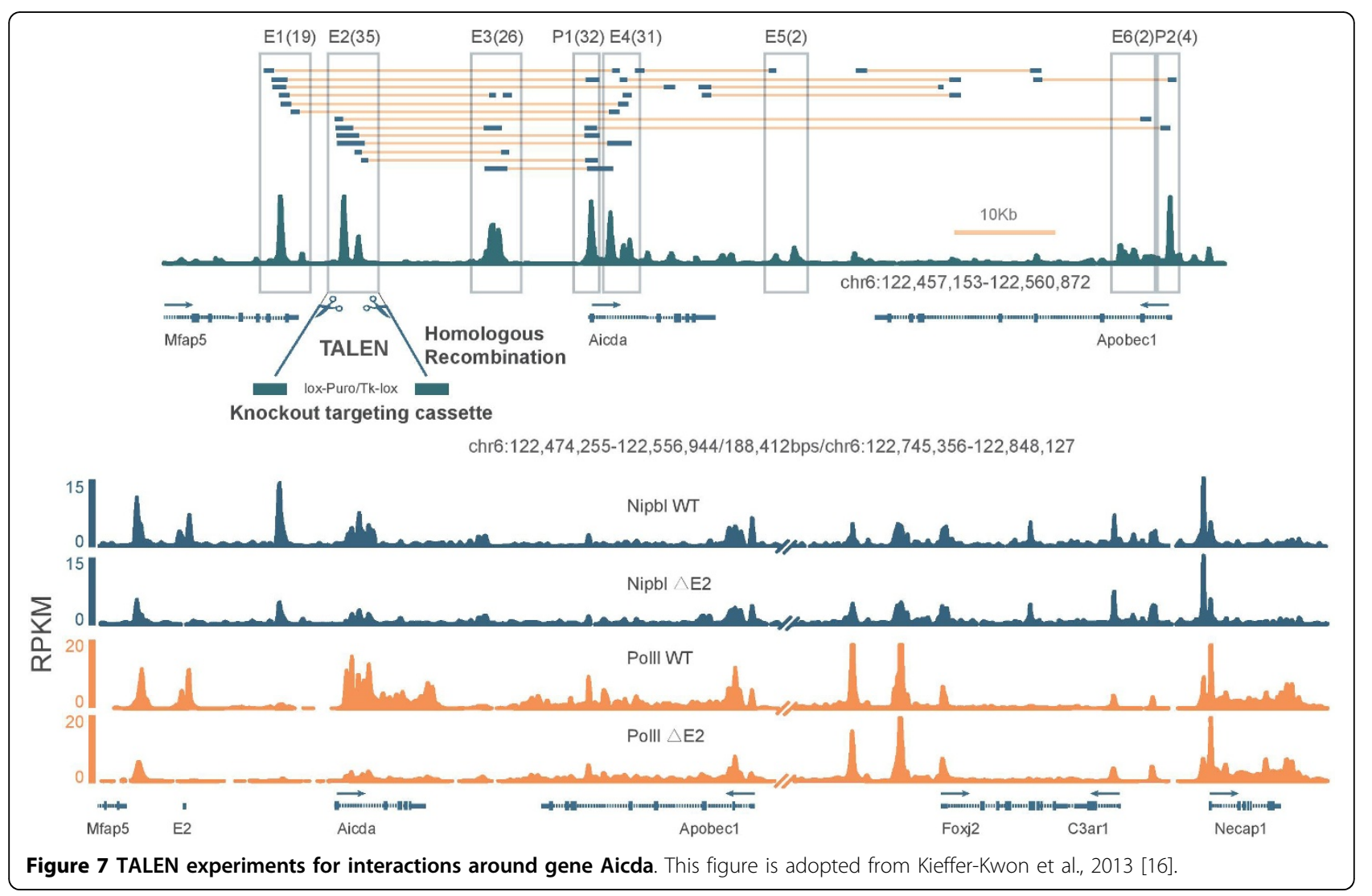


are two major approaches for modeling the 3D structure of chromatin [36]: one is physical model such as beadon-a-string model to explain the results of the experiment, and another one is the nonlinear optimization model to reconstruct the structure. Many physical properties must be taken into consideration in the first method. The first step of the nonlinear optimization model to reconstruct the structure is to convert the chromatin interaction frequency into spatial distance and then convert the spatial distance into three-dimensional structure. Of course we should do the normalization before applying the sequencing data for chromatin $3 \mathrm{D}$ structure reconstruction, because of the various biases in the experiment and the data, such as GC bias, mappability [37]. For a lack of direct parameters to evaluate the established 3D structure in a genome-wide scale, the development of electron microscopy will have a great role in the promotion of the research on 3D structure of chromatin. The visualization of chromatin interactions combined with functional assays is an important way to give people a more intuitive impression of the genome structure and a comprehensive understanding of the function of the genome.

\section{Discussion and perspectives}

ChIA-PET has been successfully applied for transcription regulation analysis in a number of studies and different chromatin interaction models have been identified. Still, there are spaces to improve the ChIA-PET protocol and analysis pipeline - to make the protocol more concise and easy to conduct, and the data analysis process more automatic and customizable.

As for the method development, the followings are several aspects to be considered.

1) Reduce the number of cells required for ChIA-PET experiments. Currently, tens of millions of cells are required in the ChIA-PET experiments. This is fine for many man-made cell lines, which could be cultured in the laboratory for more cells. However, in many real applications, like tissues from patients, there are only limited numbers of cells. To make ChIA-PET applicable to such real situations, we need to reduce the number of cells required in the experiment.

2) Make the DNA fragments longer. In the current ChIA-PET protocol, restriction enzyme MmeI is used to digest the ligated DNAs to prepare the DNA constructs, and only 20 nucleotides from the DNA fragments are kept for further analysis, which is quite short, especially for the genome with high repetitive sequence. We need to make the DNA fragment longer for more specific mapping of the reads to the reference genome.

3) Improve the comprehensiveness of the current pipeline for ChIA-PET data processing. Currently, the ChIA-PET Tool can perform the fundamental process of the ChIA-PET data. Methods for more comprehensive analysis of ChIA-PET data are not publically available. This hinders the application of ChIA-PET.

4) $3 \mathrm{D}$ genome structure reconstruction. At present, interaction data generated by ChIA-PET and $\mathrm{Hi}-\mathrm{C}$ are mainly exhibited through $2 \mathrm{D}$ graphs, which is far away from 3D visualization. We need to reconstruct the 3D structure of the genome inside the nucleus to demonstrate the chromatin interactions in the real spatial manner[38].

5) Large-scale validation of chromatin interactions and verification of their functions. Resolution of DNA-FISH needs to be enhanced to get more precise result. Observation of exquisite structure of the whole genome is still a long-term task, considering cell is in a dynamic state. Tens of thousands of chromatin interactions have already been reported by ChIA-PET, but only tens to hundreds have been validated with DNA-FISH and much more needs to be done. In addition, the functions of such interactions need to be examined one by one, with different technologies. The recent progress in genome editing technology CRISPR/Cas9 makes it a promising choice for such a task.

6) The field of transcription regulation focuses on the exploration of relationships between DNA, RNA and protein separately. We need new methods to demonstrate how these factors work together to regulate gene transcription, not just with partial information.

In addition to ChIA-PET method development, $\mathrm{Hi}-\mathrm{C}$ method and applications have shown some interesting progress. Gavrilov et al. [39] discussed variability of chromosome contacts between individual cells and suggested in-gel selection of contacting genome fragments. Thévenin et al. [40] show using $\mathrm{Hi}-\mathrm{C}$ contact map that chromosomal segments close in the $3 \mathrm{D}$ space of the nucleus tend to contain genes - members of the same functional group. It is expected that the combination of low-resolution structure from $\mathrm{Hi}-\mathrm{C}$ and high-resolution structure from ChIA-PET will inspire more insights about chromatin structures and their functions in biology.

In the near future, we hope the development of the new technologies will fulfill all the mentioned considerations and lead to novel insights about the mechanism for gene transcription regulation.

\section{Funding}

GL was supported by the Fundamental Research Funds for the Central Universities [2662014PY001]. KEV and NAK were supported by the RSF grant 14-24-00123.

\section{Additional material}

Additional file 1: List of published ChIA-PET data. 


\section{Abbreviations}

ChIP-PET: Chromatin ImmunoPrecipitation - Paired-End Tag; ChIA-Seg: Chromatin ImmunoPrecipitation with sequencing; ChIA-PET: Chromatin Interaction Analysis with Paired-End Tag sequencing; RNAPII: RNA Polymerase II.

\section{Competing interests}

The authors declare that they have no competing interests.

\section{Authors' contributions}

GL conceived and coordinated the project. GL, LC, HC, PH, and QZ wrote this manuscript, with contribution from others.

\section{Acknowledgements}

We thank to anonymous reviewers for critical remarks, A. Spitsina for technical assistance and Mr. Jianxin Li for the graphical assistance.

\section{Declarations}

Publication of this article has been funded by the Russian Science Foundation (RSF 14-24-00123).

This article has been published as part of BMC Genomics Volume 15 Supplement 12, 2014: Selected articles from the IX International Conference on the Bioinformatics of Genome Regulation and Structure \Systems Biology (BGRSISB-2014): Genomics. The full contents of the supplement are available online at http://www.biomedcentral.com/bmcgenomics/supplements/15/S12.

\section{Authors' details}

${ }^{1}$ National Key Laboratory of Crop Genetic Improvement, College of Informatics, Huazhong Agricultural University, 1, Shizishan Street, Wuhan, 430070, China. ${ }^{2}$ Institute of Cytology and Genetics SB RAS, Lavrentyeva, 10, Novosibirsk, 630090, Russia. ${ }^{3}$ Novosibirsk State University, Pirogova, 2, Novosibirsk, 630090, Russia. ${ }^{4}$ The Jackson Laboratory for Genomic Medicine, 10 Discovery Drive, Farmington, CT 06032, USA.

Published: 19 December 2014

\section{References}

1. Ren B, Robert F, Wyrick JJ, Aparicio O, Jennings EG, Simon I, Zeitlinger J, Schreiber J, Hannett N, Kanin E, Volkert TL, Wilson CJ, Bell SP, Young RA Genome-wide location and function of DNA binding proteins. Science 2000, 290(5500):2306-2309.

2. Wei $C L$, Wu Q, Vega VB, Chiu KP, Ng P, Zhang T, Shahab A, Yong HC, Fu Y, Weng Z, Liu J, Zhao XD, Chew JL, Lee YL, Kuznetsov VA, Sung WK, Miller LD, Lim B, Liu ET, Yu Q, Ng HH, Ruan Y: A global map of p53 transcription-factor binding sites in the human genome. Cell 2006, 124(1):207-219.

3. Johnson DS, Mortazavi A, Myers RM, Wold B: Genome-wide mapping of in vivo protein-DNA interactions. Science 2007, 316(5830):1497-1502.

4. Dekker J, Rippe K, Dekker M, Kleckner N: Capturing chromosome conformation. Science 2002, 295(5558):1306-1311.

5. Zhao Z, Tavoosidana G, Sjolinder M, Gondor A, Mariano P, Wang S, Kanduri C, Lezcano M, Sandhu KS, Singh U, Pant V, Tiwari V, Kurukuti S, Ohlsson R: Circular chromosome conformation capture (4C) uncovers extensive networks of epigenetically regulated intra- and interchromosomal interactions. Nat Genet 2006, 38(11):1341-1347.

6. Simonis M, Klous P, Splinter E, Moshkin Y, Willemsen R, de Wit E, van Steensel B, de Laat W: Nuclear organization of active and inactive chromatin domains uncovered by chromosome conformation captureon-chip (4C). Nat Genet 2006, 38(11):1348-1354.

7. Dostie J, Richmond TA, Arnaout RA, Selzer RR, Lee WL, Honan TA, Rubio ED, Krumm A, Lamb J, Nusbaum C, Green RD, Dekker J: Chromosome Conformation Capture Carbon Copy (5C): a massively parallel solution for mapping interactions between genomic elements. Genome Res 2006, 16(10):1299-1309.

8. de Wit $\mathrm{E}$, de Laat W: A decade of 3 C technologies: insights into nuclear organization. Genes Dev 2012, 26(1):11-24.

9. Fullwood MJ, Liu MH, Pan YF, Liu J, Xu H, Mohamed YB, Orlov YL, Velkov S, Ho A, Mei PH, Chew EG, Huang PY, Welboren WJ, Han Y, Ooi HS, Ariyaratne PN, Vega VB, Luo Y, Tan PY, Choy PY, Wansa KD, Zhao B, Lim KS, Leow SC, Yow JS, Joseph R, Li H, Desai KV, Thomsen JS, Lee YK, et al: An oestrogen-receptor-alpha-bound human chromatin interactome. Nature 2009, 462(7269):58-64.

10. Lieberman-Aiden E, van Berkum NL, Williams L, Imakaev M, Ragoczy T, Telling A, Amit I, Lajoie BR, Sabo PJ, Dorschner MO, Sandstrom R, Bernstein B, Bender MA, Groudine M, Gnirke A, Stamatoyannopoulos J, Mirny LA, Lander ES, Dekker : Comprehensive mapping of long-range interactions reveals folding principles of the human genome. Science 2009, 326(5950):289-293.

11. Li G, Ruan X, Auerbach RK, Sandhu KS, Zheng M, Wang P, Poh HM, Goh Y, Lim J, Zhang J, Sim HS, Peh SQ, Mulawadi FH, Ong CT, Orlov YL, Hong S, Zhang Z, Landt S, Raha D, Euskirchen G, Wei CL, Ge W, Wang H, Davis C, Fisher-Aylor Kl, Mortazavi A, Gerstein M, Gingeras T, Wold B, Sun $T$, et al: Extensive promoter-centered chromatin interactions provide a topological basis for transcription regulation. Cell 2012, 148(1-2):84-98.

12. Chepelev I, Wei G, Wangsa D, Tang Q, Zhao K: Characterization of genome-wide enhancer-promoter interactions reveals co-expression of interacting genes and modes of higher order chromatin organization. Cell Res 2012, 22(3):490-503.

13. Handoko L, Xu H, Li G, Ngan CY, Chew E, Schnapp M, Lee CW, Ye C, Ping JL, Mulawadi F, Wong E, Sheng J, Zhang Y, Poh T, Chan CS, Kunarso G, Shahab A, Bourque G, Cacheux-Rataboul V, Sung WK, Ruan Y, Wei CL: CTCF-mediated functional chromatin interactome in pluripotent cells. Nat Genet 2011, 43(7):630-638.

14. Dowen JM, Fan ZP, Hnisz D, Ren G, Abraham BJ, Zhang LN, Weintraub AS, Schuijers J, Lee Tl, Zhao K, Young RA: Control of cell identity genes occurs in insulated neighborhoods in Mammalian chromosomes. Cell 2014, 159(2):374-387.

15. Zhang $Y$, Wong $C H$, Birnbaum RY, Li G, Favaro R, Ngan CY, Lim J, Tai E, Poh HM, Wong E, Mulawadi FH, Sung WK, Nicolis S, Ahituv N, Ruan Y, Wei $\mathrm{CL}$ : Chromatin connectivity maps reveal dynamic promoterenhancer long-range associations. Nature 2013, 504(7479):306-310.

16. Kieffer-Kwon KR, Tang Z, Mathe E, Qian J, Sung MH, Li G, Resch W, Baek S, Pruett N, Grontved L, Vian L, Nelson S, Zare H, Hakim O, Reyon D, Yamane A, Nakahashi H, Kovalchuk AL, Zhou J, Joung JK, Sartorelli V, Wei CL, Ruan X, Hager GL, Ruan Y, Casellas R: Interactome maps of mouse gene regulatory domains reveal basic principles of transcriptional regulation. Cell 2013, 155(7):1507-1520.

17. Papantonis A, Kohro T, Baboo S, Larkin JD, Deng B, Short P, Tsutsumi S, Taylor S, Kanki Y, Kobayashi M, Li G, Poh HM, Ruan X, Aburatani H, Ruan Y, Kodama T, Wada Y, Cook PR: TNFalpha signals through specialized factories where responsive coding and miRNA genes are transcribed. EMBO J 2012, 31(23):4404-4414.

18. Demare LE, Leng J, Cotney J, Reilly SK, Yin J, Sarro R, Noonan JP: The genomic landscape of cohesin-associated chromatin interactions. Genome Research 2013, 23(8):1224-1234.

19. Heidari N, Phanstiel DH, He C, Grubert F, Jahanbanian F, Kasowski M, Zhang MQ, Snyder MP: Genome-wide map of regulatory interactions in the human genome. Genome Research 2014.

20. Sandhu KS, Li G, Poh HM, Quek YL, Sia YY, Peh SQ, Mulawadi FH, Lim J, Sikic M, Menghi F, Thalamuthu A, Sung WK, Ruan X, Fullwood MJ, Liu E, Csermely $P$, Ruan $Y$ : Large-scale functional organization of long-range chromatin interaction networks. Cell Rep 2012, 2(5):1207-1219.

21. Zhang J, Poh HM, Peh SQ, Sia YY, Li G, Mulawadi FH, Goh Y, Fullwood MJ, Sung WK, Ruan X, Ruan Y: ChIA-PET analysis of transcriptional chromatin interactions. Methods 2012, 58(3):289-299.

22. Li G, Fullwood MJ, Xu H, Mulawadi FH, Velkov S, Vega V, Ariyaratne PN, Mohamed YB, Ooi HS, Tennakoon C, Wei CL, Ruan Y, Sung WK: ChIA-PET tool for comprehensive chromatin interaction analysis with paired-end tag sequencing. Genome Biol 2010, 11(2):R22.

23. Paulsen J, Rodland EA, Holden L, Holden M, Hovig E: A statistical model of ChIA-PET data for accurate detection of chromatin 3D interactions. Nucleic acids research 2015, 42(18):e143.

24. Li H, Durbin R: Fast and accurate short read alignment with BurrowsWheeler transform. Bioinformatics 2009, 25(14):1754-1760.

25. Langmead B, Trapnell C, Pop M, Salzberg SL: Ultrafast and memoryefficient alignment of short DNA sequences to the human genome. Genome Biol 2009, 10(3):R25.

26. Tennakoon C, Purbojati RW, Sung WK: BatMis: a fast algorithm for kmismatch mapping. Bioinformatics 2012, 28(16):2122-2128. 
27. Li H, Handsaker B, Wysoker A, Fennell T, Ruan J, Homer N, Marth G, Abecasis G, Durbin R: The Sequence Alignment/Map format and SAMtools. Bioinformatics 2009, 25(16):2078-2079.

28. Quinlan AR, Hall IM: BEDTools: a flexible suite of utilities for comparing genomic features. Bioinformatics 2010, 26(6):841-842.

29. Langer-Safer PR, Levine M, Ward DC: Immunological method for mapping genes on Drosophila polytene chromosomes. Proceedings of the National Academy of Sciences of the United States of America 1982, 79(14):4381-4385.

30. He C, Wang X, Zhang MQ: Nucleosome eviction and multiple co-factor binding predict estrogen-receptor-alpha-associated long-range interactions. Nucleic acids research 2014, 42(11):6935-6944.

31. Sandhu KS, Li G, Sung WK, Ruan Y: Chromatin interaction networks and higher order architectures of eukaryotic genomes. J Cell Biochem 2011, 112(9):2218-2221.

32. Chen D, Fu LY, Zhang Z, Li G, Zhang H, Jiang L, Harrison AP, Shanahan HP, Klukas C, Zhang HY, Ruan Y, Chen LL, Chen M: Dissecting the chromatin interactome of microRNA genes. Nucleic acids research 2014, 42(5):3028-3043.

33. Hu M, Deng K, Qin Z, Dixon J, Selvaraj S, Fang J, Ren B, Liu JS: Bayesian inference of spatial organizations of chromosomes. PLOS computational biology 2013, 9(1):e1002893.

34. Peng C, Fu LY, Dong PF, Deng ZL, Li JX, Wang XT, Zhang HY: The sequencing bias relaxed characteristics of $\mathrm{Hi}-\mathrm{C}$ derived data and implications for chromatin 3D modeling. Nucleic acids research 2013, 41(19):e183.

35. Zhang Z, Li G, Toh KC, Sung WK: 3D Chromosome Modeling with SemiDefinite Programming and Hi-C Data. Journal of computational biology 2013, 20(11):831-846.

36. Peng $C$, Li G, Zhang $H$, Ruan $Y$ : Reconstruction of three-dimensional structures of chromatin and its biological implications. SCIENTIA SINICA Vitae 2014, 44:794-802.

37. Yaffe E, Tanay A: Probabilistic modeling of $\mathrm{Hi}-\mathrm{C}$ contact maps eliminates systematic biases to characterize global chromosomal architecture. Nat Genet 2011.

38. Li GL, Yuan YJ, Gu RS, et al: Emergence of 3D genomics (in Chinese). Chin Sci Bull (Chin Ver) 2014, 59:1165-1172.

39. Gavrilov AA, Chetverina HV, Chermnykh ES, Razin SV, Chetverin AB: Quantitative analysis of genomic element interactions by molecular colony technique. Nucleic acids research 2014, 42(5):e36.

40. Thevenin A, Ein-Dor L, Ozery-Flato M, Shamir R: Functional gene groups are concentrated within chromosomes, among chromosomes and in the nuclear space of the human genome. Nucleic acids research 2014, 42(15):9854-9861.

doi:10.1186/1471-2164-15-S12-S11

Cite this article as: Li et al:: Chromatin Interaction Analysis with Paired-

End Tag (ChIA-PET) sequencing technology and application. BMC Genomics 2014 15(Suppl 12):S11.

\section{Submit your next manuscript to BioMed Central and take full advantage of:}

- Convenient online submission

- Thorough peer review

- No space constraints or color figure charges

- Immediate publication on acceptance

- Inclusion in PubMed, CAS, Scopus and Google Scholar

- Research which is freely available for redistribution

Submit your manuscript at www.biomedcentral.com/submit
Biomed Central 